\title{
DAPT in the control of human hair follicle stem cell proliferation and differentiation
}

\author{
Jindou Jiang, Yong Miao, Shune Xiao, Zhidan Zhang, Zhiqi Hu
}

Department of Plastic and Cosmetic Surgery, Nanfang Hospital, Southern Medical University, China Head of Department: Jianhua Gao

Postep Derm Alergol 2014; XXXI, 4: 201-206

DOI: $10.5114 /$ pdia.2014.44002

\begin{abstract}
Introduction: Stem cells from hair follicle have great therapeutic applications in regenerative medicine as sources of cells for transplantation. The differentiation pathway selected by hair follicle stem cells (HFSC) is largely determined by local microenvironmental signals. In this study, human hair follicle stem cells were treated with Notch signaling blocker to explore a new approach to modulate human hair follicle stem cell proliferation and differentiation in vitro. Aim: To define the functional consequences of blocking the Notch signaling pathway on the proliferation and differentiation of human HSCs.

Material and methods: The human hair follicle stem cells were treated with various concentrations of Notch signaling blocker DAPT (24-diamino-5-phenylthiazole). The viability of the cells was investigated with clonogenicity assays. The expression of stem cell markers, cell cycle and cell apoptosis were analysed by flow cytometry.

Results: Notch blocking leads to promotion of human hair follicle stem cell proliferation and inhibition of differentiation in response to DAPT. The maximum effect of DAPT on the viability of human HFSC was observed at a concentration of $20 \mu \mathrm{M}$. We found that DAPT treatment results in downregulation of Hes1 and p21 and upregulation of Wnt10b.

Conclusions: $\gamma$-Secretase inhibitor DAPT has a modulatory effect on the human HFSC. The DAPT may modulate human hair follicle stem cell proliferation and differentiation through regulation of p21 and Wnt-10b.
\end{abstract}

Key words: stem cells, cell differentiation, Notch, DAPT.

\section{Introduction}

Multipotent adult stem cells have many potential therapeutic applications. Our recent findings suggest that hair follicles are a promising source of easily accessible multipotent stem cells. Hair follicle stem cells can form neurons and other cell types, and thus adult hair follicle stem cells could have important therapeutic applications. The use of hair follicle stem cells in regenerative medicine overcomes critical problems of embryonic stem cells or induced pluripotent stem cells in that the hair follicle stem cells are multipotent, readily accessible, non-oncogenic, and are not associated with ethical issues.

Stem cells from hair follicle are of interest to regenerative medicine as sources of cells for transplantation. The differentiation pathway selected by hair follicle stem cells (HFSC) is largely determined by local microenvironmental signals. In this article we discuss the diverse ways in which Notch signaling regulates hair follicle stem cell differentiation. Notch regulates keratinocyte proliferation, commitment and differentiation decisions in intact skin [1-5]. There are a number of ways in which Notch signaling can be manipulated experimentally. For example, Notch signaling can be pharmacologically reduced by $\gamma$-secretase inhibitors, which block cleavage of the Notch receptor [6]. This provides a convenient pharmacological approach to interfere with Notch function, in particular in ex vivo cell culture. In recent years, Notch pathway has been mainly monitored using transgenic animals through genetic ablation or activation. Little is known about the function of Notch signaling in human hair follicles.

Our study demonstrated that the function of Notch in human hair follicles represents an apparent contradiction to what is observed in most other cell types. In this study, we tested the hypothesis that Notch blocking in

Address for correspondence: Zhiqi Hu, Plastic and Cosmetic Surgery Department, Southern Medical University, Nanfang Hospital, Guangzhoudadaobei No. 1838, 510515 Guangzhou, China, phone: 15602332608, e-mail: huha4201587@163.com Received: 30.11.2013, accepted: 21.03.2014. 
vitro can inhibit HFSC differentiation by administration of $\gamma$-secretase inhibitors DAPT (24-diamino-5-phenylthiazole).

\section{Aim}

The aim of the study was to define the functional consequences of blocking the Notch signaling pathway on the proliferation and differentiation of human HSCS.

\section{Material and methods}

\section{Isolation and culture of human hair follicle stem cells}

Hair follicles were originally obtained in the form of transected or intact hair follicles during microscopic hair transplant procedures. The Medical Ethical Committee of the Southern Medical University approved all studies described here. The study was conducted according to the Declaration of Helsinki Principles and informed written consent was obtained from all patients. Hair follicles were derived from scalp skin of the occipital region of patients undergoing strip method hair transplant surgery. Fresh human scalp was incubated in DMEM/10\% FBS/ Dispase $(4 \mathrm{mg} / \mathrm{ml})$ for $14 \mathrm{~h}$ at $4^{\circ} \mathrm{C}$. Individual hair follicles were then extruded from tissues, and only bulge areas located between isthmus and suprabulbar regions of the hair follicles were isolated with the use of spring scissors. Collected bulge areas were attached to the dish for initia outgrowth of cells. Matrigel-coated flasks were designed for culture of HFSCS. Culture media were replaced every 3-4 days, and cells were passaged every 10 days to support maximal growth. Cells were regularly monitored for phenotype. The phenotype was evaluated by light microscopy and flow cytometry.

\section{Flow cytometry}

Flow cytometric analyses were performed. Briefly, cultured cells were blocked with $5 \%$ bovine serum albumin, and stained with a panel of antibodies including Lgr5 and Lgr6 (Biolegend, USA), or isotype controls (Biolegend, USA), respectively. The cells were subjected to flow cytometry using a BD LSRII flow cytometer and BD FACSDivaTM software.

\section{Administration of DAPT}

After 4 weeks in culture, cells were dissociated from Matrigel-coated surfaces. Matrigel was disintegrated by treatment with $1 \mathrm{mg} / \mathrm{ml}$ dispase in DMEM at $37^{\circ} \mathrm{C}$ for $30 \mathrm{~min}$, followed by aggressive mechanical trituration using Pasteur pipettes. The HFSCs were resuspended and treated with various concentrations of DAPT in a 96well plate, cells were incubated at $37^{\circ} \mathrm{C}$ in a humidified atmosphere of $5 \% \mathrm{CO}_{2}$. All cultures were performed in four types of concentrations of DAPT, in parallel: (I) $5 \mu \mathrm{M}$, (II) $10 \mu \mathrm{M}$, (III) $20 \mu \mathrm{M}$ and (IV) $40 \mu \mathrm{M}$.

\section{Colony formation assay}

The HFSCs were resuspended and treated with various concentrations of DAPT in a 96-well plate for 7 days. At end point, cells were washed twice with PBS and fixed for 5 min with $100 \%$ methanol. The HFSCs were stained with $0.2 \%$ Crystal Violet (Fisher) in $2 \%$ methanol/water $(\mathrm{v} / \mathrm{v})$. Colony number and size were determined by manual measuring.

\section{Analysis of cell cycle and apoptosis}

Fractions of HFSC were collected after treated with DAPT for 7 days. Cells were washed twice in PBS before fixation with cold $70 \%$ ethanol at $4^{\circ} \mathrm{C}$ overnight. Cells were washed twice with PBS and incubated in PBS containing $50 \mathrm{mg} / \mathrm{ml}$ propidium iodide and RNAse $(40 \mu \mathrm{g})$, for $30 \mathrm{~min}$ at $37^{\circ} \mathrm{C}$. The DNA content of each group was analyzed by flow cytometry using a FACScan, to determine the percentage of cells in various phases of the cell cycle. For apoptosis analysis, cells were incubated with FITC-conjugated Annexin V (Caltag, Burlingame, CA) for 20 min at room temperature and analyzed directly with propidium iodide by flow cytometry.

\section{Western blotting}

Cell lysate supernatants were prepared, resolved on a $7-12 \%$ sodium dodecyl sulfate bis-tris gel, and transferred to a PVDF membrane (Millipore, Billerica, MA). For Odyssey technology, the membrane was blocked with Odyssey blocking buffer and probed with anti-Hes1 antibody (1 : 1000, ab108937, abcam) and p21/CDKN1A (1 : 800, bs-0741R, Bioss), and then secondary antibody. Probed blots were scanned using an Odyssey infrared imager.

\section{Statistical analysis}

Statistical analyses were performed using Student's $t$ test, and all error bars represent the standard error of the mean.

\section{Results}

\section{Isolation and phenotypic characterization of HFSC}

The HFSC were grown from enzymatically digested human hair follicles (Figure 1). Human hair follicles were cultured for a week, round, phase-bright cells migrating from the primary culture of human hair follicle bulge areas, surface marker expression was profiled by flow cytometry. About $84.02 \%$ of the sorted cells were positive for Lgr5 expression, $66.32 \%$ of cells were positive for Lgr6 (Figure 2). These data indicate that HFSCs represent a subpopulation of cells that are positive for Lgr6, Lgr5. 

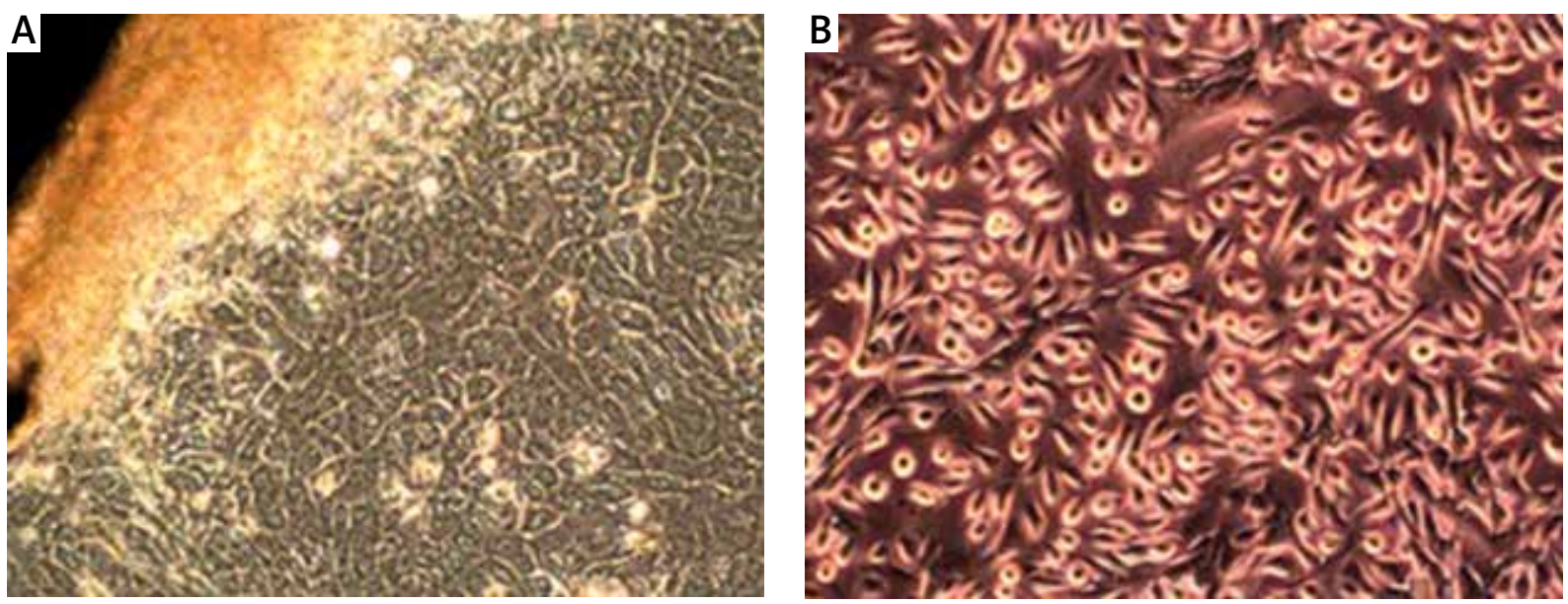

Figure 1. Cultured HFSC. A - Bulge cells expanded from explant cultures at day 7 (×100), B - Images of cultured human HFSCs cells in first passages $(\times 100)$

\section{DAPT modulate human hair follicle stem cell proliferation and differentiation}

To further characterize the morphological alteration of HFSC, we examined the colony-forming efficiency of HFSCSS after DAPT treatment. Grown at clonogenic density they show significant differences in colony formation. Cells treated with DAPT had a high self-renewal capacity and could form significantly more and larger colonies. A significant difference between $20 \mu \mathrm{M}$ DAPT group and controls is found in total colony numbers and survival fractions (Figures 3, 4; $p<0.05$ ).

To further investigate the effect of DAPT on HFSC, cell cycle analysis was shown in Figure 5. Seven days after being treated with DAPT, the percentage of cells in S-phase was $20.69 \%, 23.59 \%, 30.13 \%, 28.61 \%$, respectively. In the control group, only $19.33 \%$ of cells were in the S-phase. Our data demonstrated that Notch activity may result in cell-cycle withdrawal, causing a block of S-phase entry and the cells remain in the $\mathrm{G}_{1}$ phase (Figure 6).

To determine whether DAPT treated cells undergo apoptosis, cells were FACS analysed 7 days after DAPT administration. As shown in Figure 7, DAPT treated cells undergo a significantly lower level of apoptosis compared with control group. From the data in Figure 7, it is apparent that in all experiments, a clear decrease in the apoptosis of DAPT treated cells is seen compared with the control group, showing that blocking of N1ICD has a significant effect on the apoptosis of human hair follicle stem cell populations.

WB for the regulators indicated the effects of various concentrations of DAPT administration. Expression of cell cycle inhibitor p21 and downstream effector of Notch1 signaling Hes1 are shown in Figure 8. The DAPT results in downregulation of Hes1 and p21. Wnt-10b, uniquely among Wnts, had been upregulated after DAPT treatment.

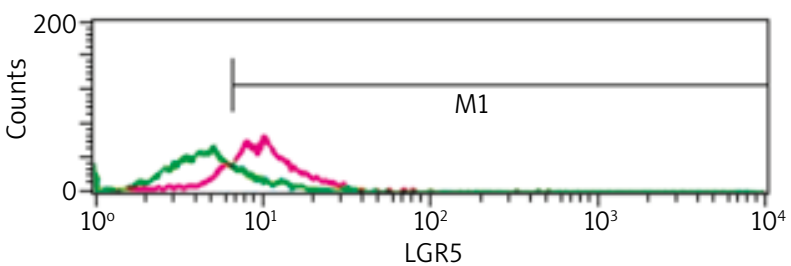

\begin{tabular}{|c|c|c|c|c|}
\hline \multicolumn{3}{|c|}{$\begin{array}{l}\text { File: 20130829BR.005 } \\
\text { Patient ID: } 5 \\
\text { Gated events: } 5280\end{array}$} & \multicolumn{2}{|c|}{$\begin{array}{l}\text { Sample ID: Laideer } \\
\text { Gate: No gate } \\
\text { Total events: } 5280\end{array}$} \\
\hline Marker & Events & $\%$ gated & $\%$ Total & median \\
\hline All & 5280 & 100.00 & 100.00 & 9.82 \\
\hline M1 & 4436 & 84.02 & 84.02 & 10.55 \\
\hline \multicolumn{3}{|c|}{$\begin{array}{l}\text { File: } 20130829 B R .001 \\
\text { Patient ID: } 1 \\
\text { Gated events: } 5175\end{array}$} & \multicolumn{2}{|c|}{$\begin{array}{l}\text { Sample ID: Laideer } \\
\text { Gate: No gate } \\
\text { Total events: } 5175\end{array}$} \\
\hline Marker & Events & $\%$ gated & $\%$ Total & median \\
\hline All & 5175 & 100.00 & 100.00 & 10.84 \\
\hline M1 & 3432 & 66.32 & 66.32 & 13.34 \\
\hline
\end{tabular}

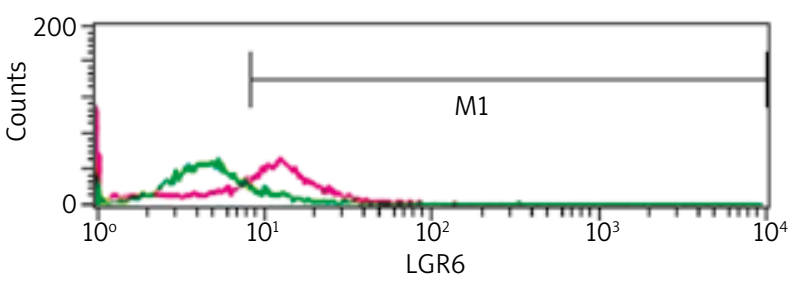

File: 20130829BR.001

Patient ID: 1

D: Laideer

Gate: No gate

Figure 2. Flow-cytometry analyses of stem cell marker expression 


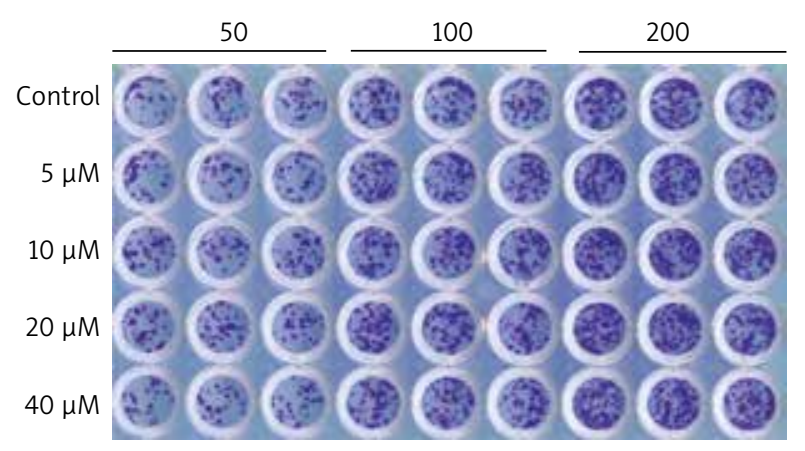

Figure 3. Colony-unit formation assay after different concentrations of DAPT treatment. Grown at clonogenic density they show significant differences in colony formation. A significant difference between $20 \mu \mathrm{M}$ DAPT group and controls is found in total colony numbers $(p<0.05)$

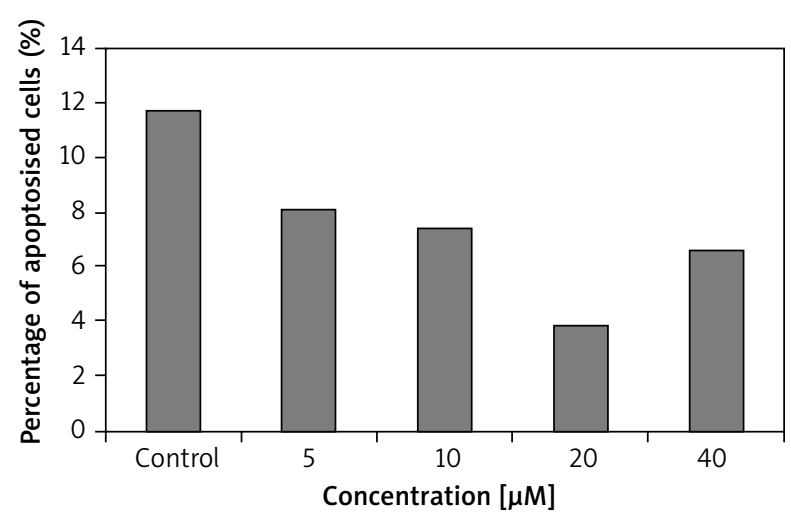

Figure 5. Effects of various concentrations of DAPT on the cell apoptosis in human HFSCs. A clear decrease in the apoptosis of DAPT treated cells is seen compared with the control group

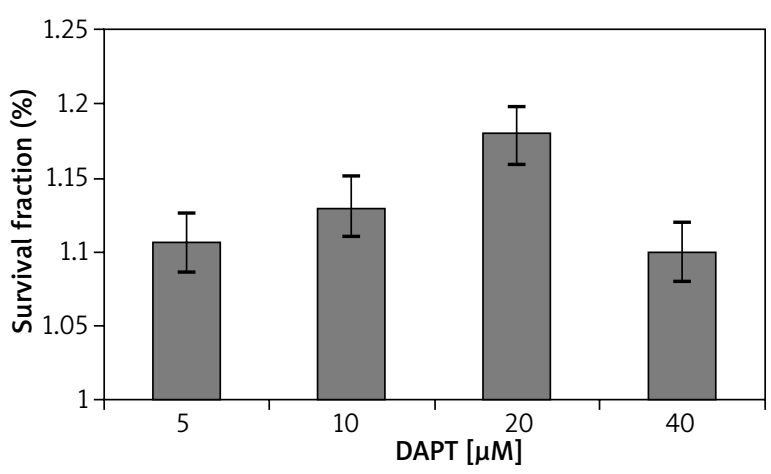

Figure 4. Effects of various concentrations of DAPT on the survival fractions of human HFSCs. Survival fraction = (colony-forming efficiency of DAPT treated group)/(colonyforming efficiency of the control group)

\section{Discussion}

The mammalian hair follicle has a stem cell niche called the 'bulge' that acts as a reservoir of multipotent stem cells. Clonal and in vivo lineage analyses of bulge cells, coupled with reconstitution assays, revealed that these undifferentiated cells are able to self-renew and contribute to all epithelial lineages in the skin, including the HF, sebaceous gland, and interfollicular epidermis. Among many reports that described various biomarkers for identifying hair follicle stem cells, the most convincing evidence was provided in recent reports suggesting that Lgr5 and Lgr6 are biomarkers for human follicular stem cells that reside in the bulge. In telogen HF, the L $g 5^{+}$cells were shown to reside exclusively in the lower bulge and hair germ [7]. These cells were shown to
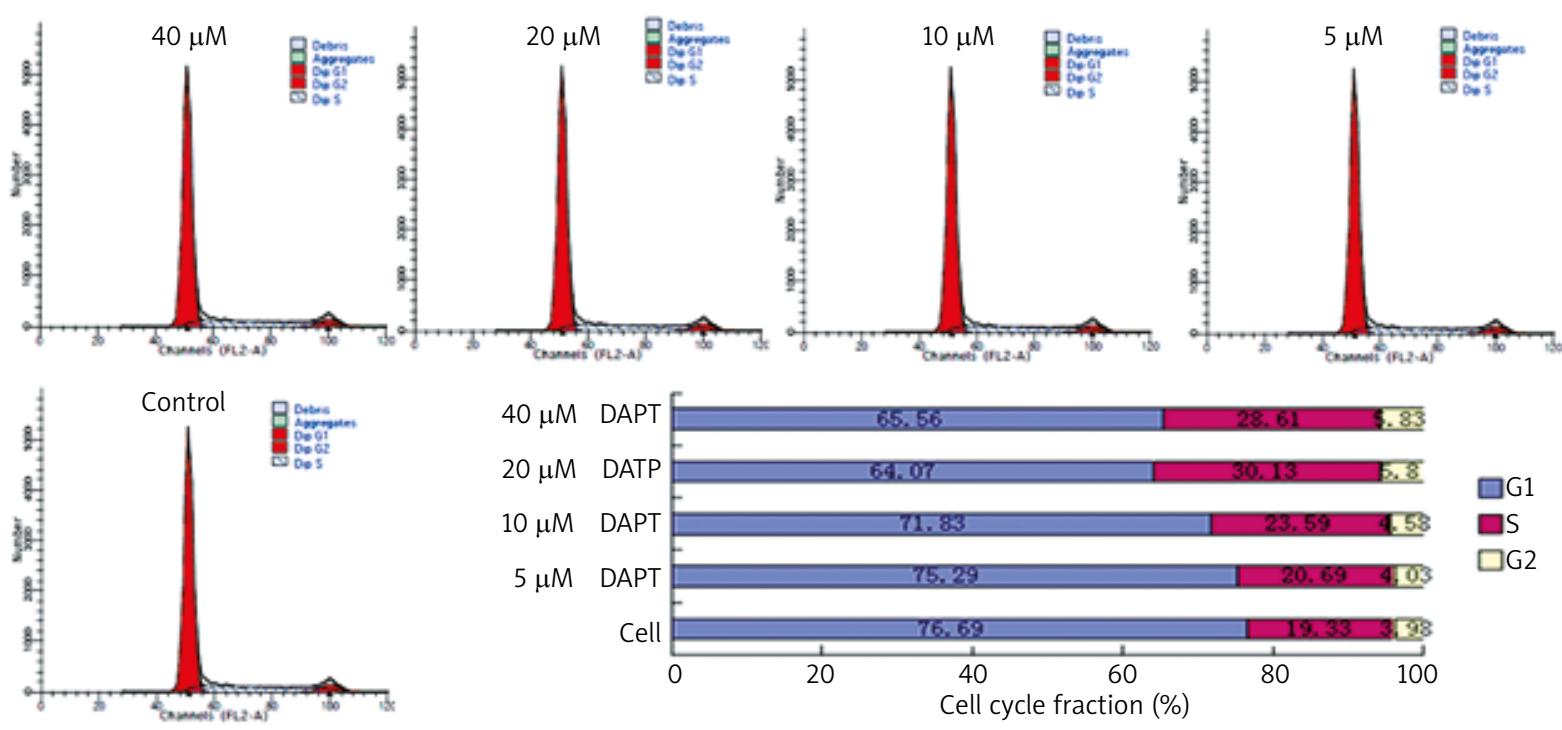

Figure 6. Cell cycle analyses of human HFSCs after DAPT treatment. The percentage of cells in S-phase was $20.69 \%$, $23.59 \%, 30.13 \%, 28.61 \%$, respectively. In the control group, only $19.33 \%$ of cells were in the S-phase 


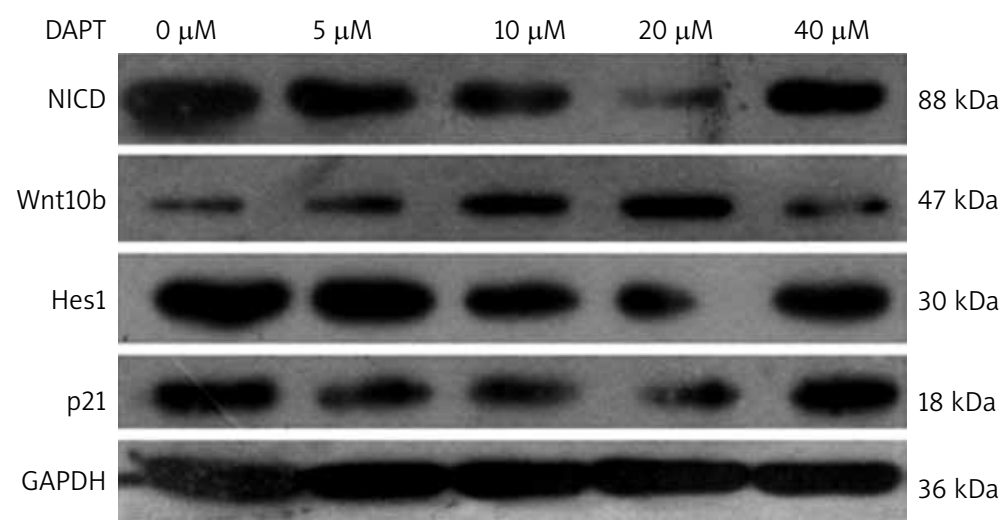

Figure 7. Expression of the regulators indicated in DAPT treated cells as determined by western blotting. Expression of cell cycle inhibitor $p 21$ and downstream effector of Notch1 signaling Hes1 are downregulated. DAPT results in upregulation of Wnt-10b

actively proliferate (at least during the anagen phase of the HF), establishing the $\mathrm{Lgr} 5^{+}$pool as a separate population to the label-retaining stem cells present in the upper bulge regions. Following transplantation, $\operatorname{Lg} \mathrm{S}^{+}$cells were capable of generating HFs [7]. A recent study identified Lgr6 as a marker for a distinct population of stem cells giving rise to all lineages of the skin [8]. Unlike the Lgr5 gene, Lgr6 is controlled by Wnt signaling [9]. This is in agreement with the notion that the active hair lineage in the lower bulge requires Wnt signaling, whereas the sebaceous and epidermal lineages are Wnt-independent [9]. A Wnt-independent Lgr6 stem cell pool can renew sebaceous cells and seed the epidermis throughout life, whereas a Wnt-dependent Lgr5 stem cell pool derives from the Lgr6 pool early in life but then becomes relatively independent.

Our studies demonstrated that after cultured for a few months, HFSC were shown to down-regulate Lgr6 expression, and were positive for the K10, indicating their commitment to differentiation consistent with their decreased growth capacity in vitro. We next sought to determine whether the human HFSCs proliferation and differentiation could be modulated by Notch signaling. Our data suggested that the function of Notch in hair follicle represents an apparent contradiction to what is observed in most other cell types. Differentiation of HFSC is promoted rather than blocked by Notch signaling. This is in line with findings that Notch has a tumor suppressor function in the skin, as mice lacking Notch1 expression in keratinocytes frequently develop tumors [10]. The Notch1 signaling pathway is evolutionarily conserved from invertebrates to vertebrates and is involved in cell-fate decisions during development [11-14]. Notch1 signaling is activated via juxtacrine binding of an adjacent cell's Delta-like or Jagged ligands with the Notch receptor. On activation, the Notch1 intracellular domain (N1-ICD) is released from the membrane receptor via enzymatic cleavage. The N1-ICD can function as a transcriptional activator, translocating into the nucleus and forming a DNA-binding complex with J kappa-recombining binding protein (RBPJ) to control the downstream effectors of Notch1 signaling, such as Hes1 [15].

Notch signaling is important in regulating hair follicle stem cell proliferation and differentiation. In our data, WB for expression of regulators and analysis of the cell cycle indicated that Notch activity may result in cell-cycle withdrawal and differentiation by upregulation of $p 21$, resulting in a block of $S$-phase entry and the cells remain in the $G$ phase (Figures 5 and 7 ). A recent study has reported that the $p 21$ promoter contains binding sites for CSL, implicating p21 as a direct target of Notch ICD/CSL. In summary, the increase in $p 21$ may inhibit the mitotic activity. Notch-induced expression of $p 21$ also requires the regulation of Notch target gene Hes1 [16, 17]. A recent link between Wnt and Notch signaling was demonstrated in keratinocytes where the tumor-suppressor function of Notch was associated with downregulation of Wnt signaling. This was achieved, at least in part, by suppression of Wnt4 gene expression by Notch target $p 21$ [18]. In hair follicle in postnatal skin, the expressions of Wnts have been reported, but only Wnt-10b showed evident promotion of epithelial cell differentiation and hair shaft growth, in contrast to Wnt3a, 5a, and 11. Our results suggest that the regulation of Wnt-10b is unique and plays an important role in differentiation of hair follicle stem cells after DAPT treatment. The combination of direct regulation of Wnt-10b and decreased activity of $p 21$ may lead to the observed modulation of HFSC proliferation and differentiation process.

The current generation of $\gamma$-secretase inhibitors has unwanted side effects, including intestinal goblet cell metaplasia in experimental animals, which precludes long-term clinical use [19]. More local and short-term administration may however be acceptable from the side 
effect standpoint, and has been contemplated for potential treatment of Notch-induced cancers [20]. This may potentially be used to locally modulate stem cell differentiation in vivo, in the context of regenerative medicine in the future.

\section{Conclusions}

Notch signaling is important in regulating hair follicle stem cell proliferation and differentiation. Notch blocking leads to promotion of human hair follicle stem cell proliferation and inhibition of differentiation in response to DAPT. $\gamma$-Secretase inhibitor DAPT has a modulatory effect on the human HFSCS. DAPT may modulate human hair follicle stem cell proliferation and differentiation through regulation of $\mathrm{p} 21$ and Wnt-10b.

\section{Acknowledgments}

This work was supported by the Natural Science Foundation of China (Grant No. 31170949). The funders had no role in study design, data collection and analysis, decision to publish, or preparation of the manuscript.

\section{Conflict of interests}

There is no conflict of interests.

\section{References}

1. Blanpain C, Lowry WE, Pasolli HA, Fuchs E. Canonical notch signaling functions as a commitment switch in the epidermal lineage. Genes Dev 2006; 20: 3022-35.

2. Lee J, Basak JM, Demehri S, Kopan R. Bi-compartmental communication contributes to the opposite proliferative behavior of Notch1-deficient hair follicle and epidermal keratinocytes. Development 2007; 134: 2795-806.

3. Pan Y, Lin MH, Tian X, et al. Gamma-secretase functions through Notch signaling to maintain skin appendages but is not required for their patterning or initial morphogenesis. Dev Cell 2004; 7: 731-43.

4. Rangarajan A, Talora C, Okuyama R, et al. Notch signaling is a direct determinant of keratinocyte growth arrest and entry into differentiation. EMBO J 2001; 20: 3427-36.

5. Cichorek M, Wachulska M, Stasiewicz A, Tymińska A. Skin melanocytes: biology and development. Postep Derm Alergol 2013; 30: 30-41.

6. Zhao LD, Guo WW, Lin C, et al. Effects of DAPT and Atoh1 overexpression on hair cell production and hair bundle orientation in cultured organ of Corti from neonatal rats. PLoS One 2011; 6: e23729.

7. Jaks V, Barker N, Kasper M, et al. Lgr5 marks cycling, yet long lived, hair follicle stem cells. Nat Genet 2008; 40: 1291-9.

8. Snippert HJ, Haegebarth A, Kasper M, et al. Lgr6 Marks stem cells in the hair follicle that generate all cell lineages of the skin. Science 2010; 327: 1385-9.

9. Fuchs E, Horsley V. More than one way to skin. Genes Develop 2008; 22: 976-85.

10. Nicolas M, Wolfer A, Raj K, et al. Notch1 functions as a tumor suppressor in mouse skin. Nat Genet 2003; 33: 416-21.
11. High FA, Zhang M, Proweller A, et al. An essential role for Notch in neural crest during cardio vascular development and smooth muscle differentiation. J Clin Invest 2007; 117: 353-63.

12. Dyer LA, ML Kirby. The role of secondary heart field in cardiac development. Dev Biol 2009; 336: 137-44.

13. Yang K, Doughman YQ, Karunamuni G, et al. Expression of active Notch1 in avian coronary development. Dev Dyn 2009; 238: $162-70$.

14. Grieskamp T, Rudat C, Ludtke TH, et al. Notch signaling regulates smooth muscle differentiation of epicardium-derived cells. Circ Res 2011; 108: 813-23.

15. Murata J, Ohtsuka T, Tokunaga A, et al. Notch-Hes1 pathway contributes to the cochlear prosensory formation potentially through the transcriptional down-regulation of p27Kip1. J Neurosci Res 2009; 87: 3521-34.

16. Mammucari C, di Vignano AT, Sharov AA, et al. Integration of Notch 1 and calcineurin/NFAT signaling pathways in keratinocyte growth and differentiation control. Dev Cell 2005; 8: 665-76.

17. Lefort K, Dotto GP. Notch signaling in the integrated control of keratinocyte growth/differentiation and tumor suppression. Semin Cancer Biol 2004; 14: 374-86.

18. Devgan V, Mammucari C, Millar SE, et al. p21WAF1/Cip1 is a negative transcriptional regulator of Wnt4 expression downstream of Notch1 activation. Genes Dev 2005; 19: 1485-95.

19. Milano J, McKay J, Dagenais C, et al. Modulation of notch processing by gamma-secretase inhibitors causes intestinal goblet cell metaplasia and induction of genes known to specify gut secretory lineage differentiation. Toxicol Sci 2004; 82: 341-58.

20.Van Es JH, Clevers H. Notch and Wnt inhibitors as potential new drugs for intestinal neoplastic disease. Trends Mol Med 2005; 11: 496-502. 